\section{Future of Wearable Health Devices: Smartwatches VS Smart Headphones}

\author{
Ali Tavakoli Golpaygani*๑, Ali Reza Mehdizadeh²
}

$\mathrm{T}$

oday's, the global life expectancy is increasing due to many factors, but the significant improvements in healthcare systems, and personal and environmental hygiene growing are the most important. In near future, we will be encountered with a significant need of social welfare and healthcare for this enormous growing aging population. Thus, it is a necessity to develop and implement new strategies and technologies to provide more efficient and better health care services with an affordable cost and less limited access among people especially with the aging population. Remote healthcare monitoring is introduced as a key solution to provide an efficient and cost-effective way instead of on-site clinical monitoring in hospitals and medical centers.

The revolution technology in electronic and digital communication is an ability to design more reliable and adaptable wearable health devices for continuous monitoring a wide range of different physiological sign and activities in real-time and from distant. The new technology capabilities with the advantage of miniaturization improves human-machine connectivity with less disconformity during normal daily life activities (at work, at home, at sport, etc.). This technology could have a significant effect on health status awareness and quality care improvement via a better support in medical diagnosis and treatment [1].

The Wearable Medical Devices Market is growing rapidly, according to the latest medical market research, the market size is exceeded USD 25 billion in 2020 and is expected to grow at over $22.9 \%$ CAGR (Compound Annual Growth Rate) from 2021 to 2027. During COVID-19, this market has experienced increased demand by increasing focus on health management systems, daily health tracking and remote healthcare monitoring. Changing lifestyle will have an intensive effect on boosting personal health and fitness products demand.

The main requirements in the concept design of a wearable health device are power, efficiency, privacy, security, reliability, connectivity, usability, portability, robustness, stability, multimodality, modifiability, maintainability, comfortable and ergonomics features.

As a general feature, the present wearable health devices could monitor some physiological vital signs including heart rate, ECG, pulse oximeters, blood pressure, breath rate, and body temperature. These parameters indicate the overall health status of a person and have tremendous diagnostic value. For the foreseeable future, with the development of a miniaturized smart sensors, it is expected the wearable health devices to be complemented with chemical sensors that will provide continuous non-invasive chemical and metabolic measurements for better medical monitoring (e.g. in sweat for diabetes patients, pharmacokinetics process in the body, metabolic diseases, and others).

For a reliable health monitoring system, the wearable health devices must be accurate and act reliable under multiple environmental conditions and activity
${ }^{1} \mathrm{PhD}$, Graduate School of

Health, University of Tech-

nology of Sydney, Sydney,

Australia

${ }^{2} \mathrm{MD}, \mathrm{PhD}$, Editor-in-Chief of

the Journal of Biomedical

Physics and Engineering,

Shiraz University of Medical

Sciences, Shiraz, Iran

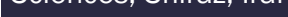

*Corresponding author: Ali Tavakoli Golpaygani Graduate School of Health, University of Technology of Sydney, Sydney, Australia E-mail: ali.tavakoligolpaygani@uts.edu.au

Received: 8 September 2021 Accepted: 12 September 2021 
situations. An accurate measurements and analysis would be vital in a health monitoring system and any noise and artifacts in this process could be resulted of many challenges in the right estimation and early detection of health abnormalities like as heart failure diseases including Atrial Fibrillation [1].

The Smartwatches and smart wristbands, as a merge of technology and fashion beyond showing time, are one of the latest developments in wearable health devices technology. A Google market trend analysis showed a tremendous increase in searches for 'smartwatch' and the huge amounts of related apps offered for them, these apps give more usability to users such as access to emails, messaging, e-banking, and much more.

One of the important concepts in the designing of wearable devices is that they should be lightweight, easy to wear and handle, long-term monitoring capability, and well sensitivity to noise and motion artifacts. Despite the smartwatches and wristbands' uses are growing rapidly, but some functional difficulties may experience based on application sites on the wrist skin, due to fragile skin, displaced and poor attachment problems and motion artifacts [2].

Recently, many of the research studies have shown a wide interest for using hearables instruments as a kind of wearable health devices for physiological parameters measurement and monitoring. The capability of this group of devices, for an accurate physiological measurement in multiple conditions, is potentially able to satisfy commercial and research-oriented markets [3].

The ear canal, because of the close position to the core of the body, is a suitable site for measuring vital physiological parameters with reliable, accurate signal quality, and more stable with a fast response in different measurement conditions. The ear canal provides a good fixation condition for hearable devices and makes a good long-term monitoring position in daily-life activities. This area also provides a protected place from the impact of extreme changing environmental conditions (Temperature, Sunlight) and movement dislocation [3].

Some studies have been shown and analyzed the suitability of the ear canal as a reliable measuring site in different physiological and environmental conditions. It has shown that the ear canal is a reliable site for measuring body temperature and pulse oximetry $\left(\mathrm{SpO}_{2}\right)$, because of a good blood flux (PPG signal quality) and less motion artifacts [4].

Although the application of Smart headphones, as hearable devices, needs more investigation in challenging environmental conditions and intense exercise activities, but the hearable PPG devices would be a strongly widespread wearable sensors technology in near future. This outstanding measurement technique would become a common readily available solution for Heart Rate (HR), Heart Rate Variability (HRV), Blood Pressure (BP), Blood Oxygen Saturation $\left(\mathrm{SpO}_{2}\right)$, and body temperature monitoring during daily living, sports, exercise and physical activities, occupational health recording, and telediagnosis.

\section{Conflict of Interest}

None

\section{References}

1. Habibzadeh H, Dinesh K, Shishvan OR, Boggio-Dandry A, Sharma G, Soyata T. A survey of healthcare Internet of Things (HIOT): A clinical perspective. IEEE Internet Things J. 2020;7(1):53-71. doi: 10.1109/jiot.2019.2946359. PubMed PMID: 33748312. PubMed PMCID: PMC7970885.

2. Auepanwiriyakul C, Waibel S, Songa J, Bentley P, Faisal AA. Accuracy and Acceptability of Wearable Motion Tracking for Inpatient Monitoring Using Smartwatches. Sensors. 2020;20(24):7313. doi: 10.3390/s20247313. PubMed PMID: 33352717. PubMed PMCID: PMC7766923.

3. Masè M, Micarelli A, Strapazzon G. Hearables: new perspectives and pitfalls of in-ear devices for physiological monitoring. A scoping review. Front Physiol. 2020;11:68886. doi: 10.3389/fphys.2020.5688866. PubMed PMID: 33178038. PubMed PMCID: PMC7596679.

4. Goverdovsky V, Von Rosenberg W, Nakamura T, Looney D, Sharp DJ, Papavassiliou C, Morrell MJ, Mandic DP. Hearables: Multimodal physiological in-ear sensing. Sci Rep. 2017;7(1):6948. doi: 10.1038/s41598-017-06925-2. PubMed PMID: 28761162. PubMed PMCID: PMC5537365. 